DOI: http://dx.doi.org/10.18203/2320-1770.ijrcog20162971

Research Article

\title{
Vault prolapse - a decade's experience
}

\section{Srinivas Krishna Jois*, Lavanya Gurumurthy Rao}

Department of Obstetrics and Gynaecology, Bangalore Medical College and Research Institute, Bangalore, Karnataka, India

Received: 27 June 2016

Accepted: 05 August 2016

\section{*Correspondence:}

Dr. Srinivas Krishna Jois,

E-mail: srinivaskjois@gmail.com

Copyright: (c) the author(s), publisher and licensee Medip Academy. This is an open-access article distributed under the terms of the Creative Commons Attribution Non-Commercial License, which permits unrestricted non-commercial use, distribution, and reproduction in any medium, provided the original work is properly cited.

\begin{abstract}
Background: Vault prolapse although an uncommon condition is an annoying problem to the patient often requiring repeat surgery for relief. The objective of this study was to study the clinical parameters of patients of vault prolapse, predisposing factors, pre-op and post-op symptoms, treatment provided and outcomes of the same over ten years at Vani Vilas Hospital, Bangalore Medical College.

Methods: A retrospective study was conducted on all patients admitted with vault prolapse and treated for the same from 2005 to 2014. Data was collected and analyzed.

Results: commonest symptom associated was mass per vagina with difficulty in voiding urine (80\%). Straining at stools and sexual dysfunction was seen in fewer than $30 \%$. 64\% of our patients had TAH and the mean duration before symptoms was 12 years (11.7 years). Majority of our patients underwent sacrospious fixation (42\%) while $24 \%$ underwent traditional repair. Abdominal sacrocolpopexy was performed on 8 patients. Intra-op complications of haemorrhage seen in 1, ureteric injury in 2 and injury to rectum in 1 patient. ICU admission was required for $5.80 \%$ of the patients were available for follow up at 2 weeks and 6 weeks and $>90 \%$ expressed subjective improvement in symptoms. Only one underwent re operation at 3 months for failure.

Conclusions: Vault prolapse is not an uncommon condition following hysterectomy especially if it was done at a young age. Careful selection of patients, measures to prevent this condition during primary hysterectomy is the need of the hour.
\end{abstract}

Keywords: Vault prolapse, Sacrospinous fixation, Sacrocolpopexy

\section{INTRODUCTION}

As the general longevity is improving, pelvic floor disorders are becoming more prevalent in women. Life time risk of surgery for pelvic organ prolapse (POP) or incontinence is estimated to be $11 \%$ with a reoperation rate of $29 \%$ for failure. ${ }^{1}$ Over the next 30 years the demand for treatment of POP is expected to increase to $45 \%$ as the number of women over 50 years increases. ${ }^{2}$ The increasing aging population also expects a good quality of life and sexual satisfaction. There is also an increase in number of women undergoing hysterectomy at a young age and hence at risk of complications including vault prolapse (VP).
The vaginal vault is the expanded proximal portion of the vaginal canal. Post hysterectomy vaginal vault prolapse is defined as descent of the vaginal cuff scar below a point that is $2 \mathrm{~cm}$ less than total vaginal length above the plane of hymen by the International Incontinence Society. Although it is not a common condition occurring only in about $1 \%$ of hysterectomies, the incidence is definitely on the rise, as much as five fold, thanks to the extended longevity of women.

Better understanding of pelvic anatomy, architecture and dynamics has changed the treatment modalities over the years. Several surgical procedures have been described, 
but randomised controlled studies specifically addressing post-hysterectomy vaginal vault prolapse are limited.

The traditional vaginal repair of the vault prolapse involved anterior and posterior colporrhaphy and perineorrhaphy often resulting in much narrowing of the vaginal canal and the introitus. The adequacy of standard repair operation with anterior and posterior vaginal wall repair and enterocele closure has been questioned. It does not support the vaginal vault and risks vaginal narrowing and shortening, and thus dyspareunia in nearly $20 \%$, especially when posterior repair is carried out. ${ }^{3}$

Surgery for VP now aims to suspend the vaginal vault to existing pelvic support structures or anchors. Sacrospinous fixation and abdominal colposuspension have equal efficacy and relative benefits and risks as demonstrated by Maher et al in their RCT. ${ }^{4}$

In the 1990s, the risk of reoperation after primary standard vaginal procedure was estimated to be as high as $30 \%$ to $50 \%$. In order to reduce the risk of relapse, gynaecological surgeons started to use mesh implants in pelvic organ reconstructive surgery with the emergence of new complications. Recent studies have nevertheless shown that the risk of POP recurrence requiring reoperation is lower than previously estimated, being closer to $10 \%$ rather than $30 \% .^{5}$ Obliterative procedures are highly effective interventions but are reserved for women unfit for other procedures.

The objective of this study was to study the clinical parameters of patients presenting with vault prolapse and the treatment offered over 10 years in Vani Vilas Hospital attached to Bangalore Medical College and Research Institute. And to study the relationship between the type of hysterectomy leading to Vault Prolapse, its indication and the duration after which symptomatic vault prolapse occurred. To study the immediate complications and outcome of different surgical treatment modalities. The specific aims of the study were to investigate operative and postoperative complications, perceived health, sexual activity, urinary symptoms, and patient satisfaction with different surgical treatment modalities.

\section{METHODS}

A retrospective study was conducted on all patients admitted with vault prolapse and treated for the same in Vani Vilas Hospital (VVH) a tertiary referral hospital attached to Bangalore Medical College over a period of 10 years from 2005 to 2014. Data was collected from patients, case sheets, OT registers and other hospital records. Patient's age, parity, symptomatology, clinical examination findings like degree of vault prolapse according to Shaw's classification (POP Q system not used as it is being used in the institution only recently), other associated features like incontinence, pain, discharge etc. were noted. The details of previous hysterectomy surgery, the reason for the same, the interval between hysterectomy and development of vault prolapse and any pre disposing factors like chronic cough, obesity, advanced age, surgical complications etc. were studied. The treatment modalities that were offered for vault prolapse in VVH over the years and the outcome of the treatment including intra and post-op complications were analyzed. Some of the patients were followed for 6 weeks to 6 months.

\section{RESULTS}

A total of 56 patients' records were reviewed and following observations were made. The incidence of vault prolapse among the gynaecology surgeries in the hospital was about 1 in 200.

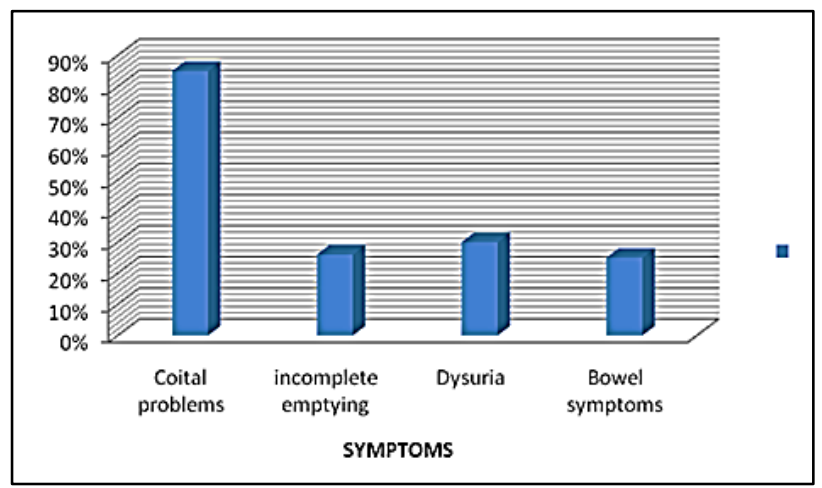

Figure 1: Symptomatology.

$70 \%$ of patients were beyond 50 years of age. The median age of vault prolapse patients in our study was 55 years (ranging from 35 years to 75 years). $54 \%$ of our patients had history of undergoing abdominal hysterectomy and the remaining had had vaginal hysterectomy. There were no cases of laparoscopic hysterectomy. Mean interval between hysterectomy and seeking of treatment for vault prolapse was 11.8 years with a range from 2 years to 25 years.

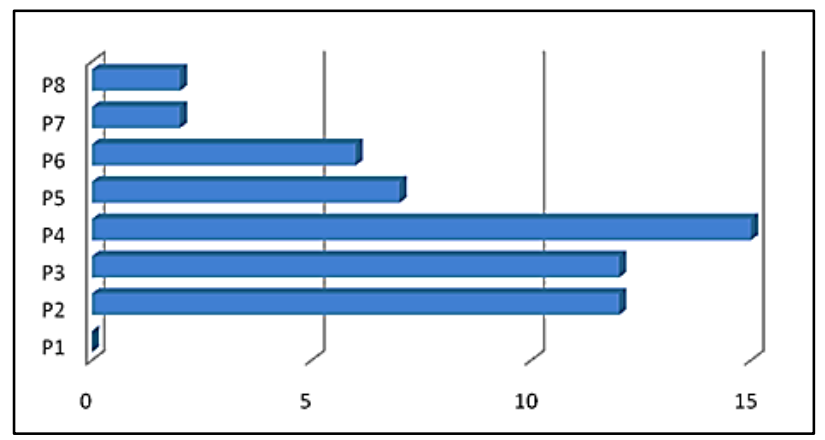

Figure 2: Parity distribution.

Most common indications for previous abdominal hysterectomy was abnormal uterine bleeding, fibroid uterus as deduced from history given by the patients. Among the 26 patients who underwent vaginal hysterectomy nearly 20 of them had complaints of mass 
per vagina and of the remaining 6 patients there was no documentation about the uterine descent during vaginal hysterectomy. Documentation regarding the presence of descent at the time of abdominal hysterectomy too was not available.

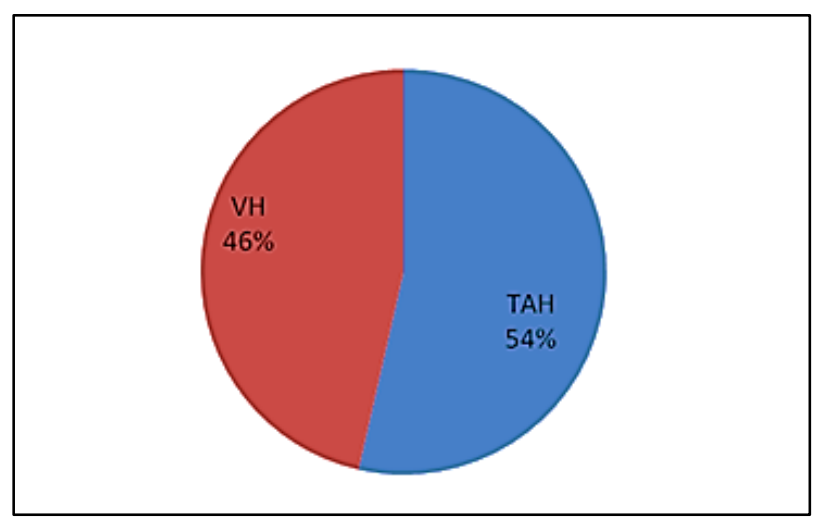

Figure 3: Route of previous hysterectomy.

All the patients in the present study were parous women and the parity ranged from 2 to 8 , median being 4 . Only one patient had caesarean delivery. All the rest had had vaginal deliveries.

Symptom analysis revealed the following. Apart from mass per vaginum, majority of patients had voiding problems which included difficulty in voiding and incomplete evacuation (85\%). Pain and dysuria was noted in 15 patients $(26 \%)$. Coital problem was a complaint in $30 \%$ of patients who were sexually active. Twelve (12) patients complained of constipation, and sense of incomplete bowel evacuation. Anal incontinence was seen in 2 patients.

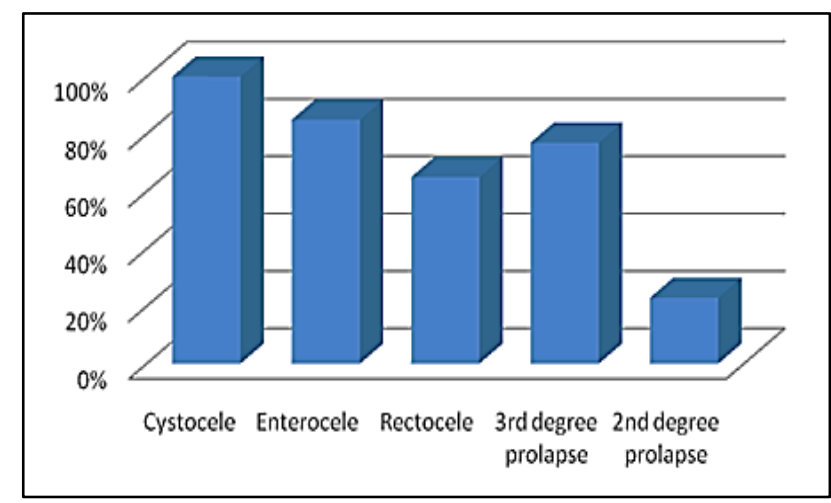

Figure 4: Examination findings.

Some of the possible pre disposing factors that were identified in our study included chronic cough (in 3 patients) which possibly resulted in raised abdominal pressures, surgery performed by non-gynaecologists in which case proper understanding of female pelvic support systems and restoration of its strength was probably lacking and lastly advanced age with hypo estrogenic state leading to loss of tissue strength.
On examination $22 \%$ of the patients were hypertensives, $31 \%$ were diabetics, $36 \%$ were with BMI $>25$ and $10 \%$ had a BMI of $<18.10 \%$ were anaemic. All the patients had mass per vaginum either $2^{\text {nd }}$ degree or $3^{\text {rd }}$ degree (23\% and $77 \%$ ). Cystocele was the most common finding and was seen in all the 56 patients. Enterocele was seen in $85 \%$ of the patients and rectocele in a total of $65 \%$ of the patients.

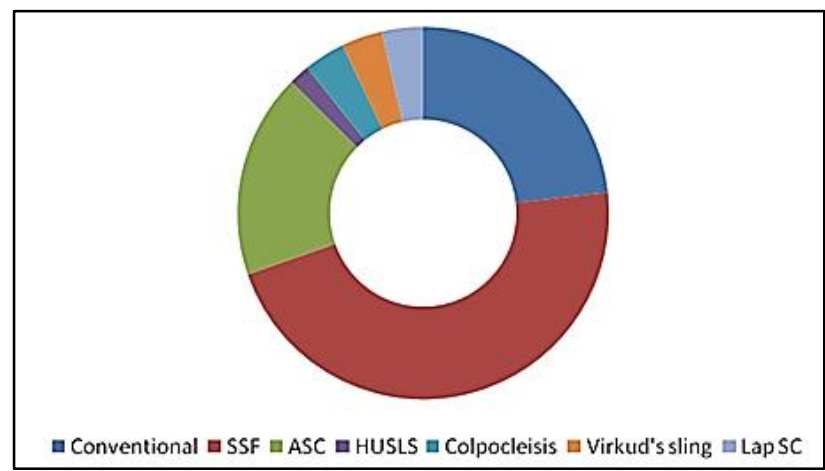

Figure 5: Type of surgery done.

All our patients were evaluated clinically for urinary incontinence which was found to be present in only $4 \%$ of the patients. $57 \%$ of the patients had grossly decreased levator tone and widely gaping introitus. Anal sphincter tone was normal in all the patients.

Initial part of the study period, the first 5 to 6 years has seen most of the cases undergoing traditional repair, i.e., anterior colporrhaphy and posterior colpoperineorrhaphy with a few cases undergoing other procedures. Conventional repair was done in 13 patients. Included cystocele repair in all and rectocele and pelvic floor repair in 6 patients.

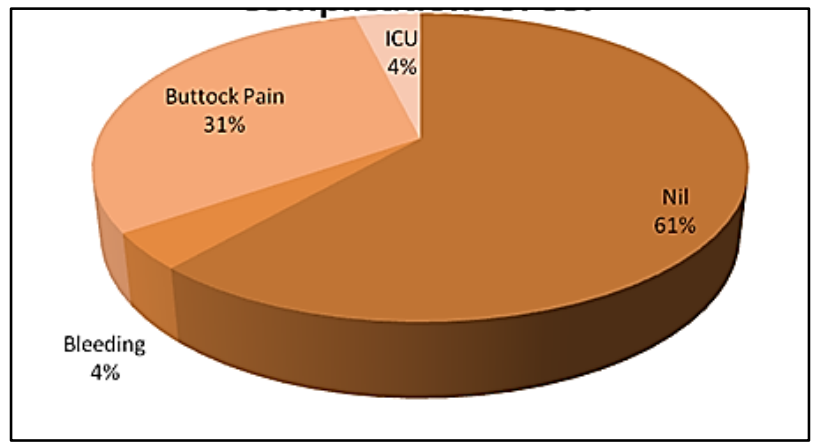

Figure 6: Complications of sacrospinous fixation.

Right sacrospinous fixation: fixing the vaginal vault to the sacrospinous ligament using permanent suture material like prolelne was the most common procedure performed in the study (26 patients, $46 \%$ ).

Abdominal sacrocolpopexy (ASC) involves apical suspension of the vault with a permanent mesh fixed to the anterior longitudinal ligament of the sacrum; 
typically, the mesh is attached to the anterior and posterior aspects of the vault with possible 'mesh extension' to correct prolapse in other compartments which was done in 10 patients $(18 \%)$ in our study.

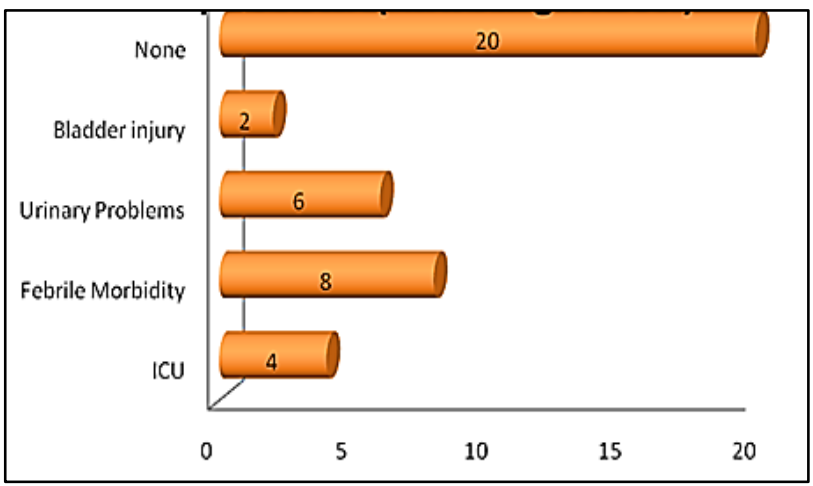

Figure 7: Complications of other surgeries.

High uterosacral ligament fixation HUSLS can be done vaginally, abdominally or laparoscopically and involves bilateral suspension of the vaginal vault, using sutures, to the uterosacral ligaments near the level of the ischial spine. This was done in only 1 patient vaginally during the study period of 10 years as a part of a workshop.

Several other procedures were offered occasionally after analysing and individualising treatment: colpocleisis was done in 2 patients both in their 70s. Virkud's sling procedure was performed in two patients, and laparoscopic sacrocolpopexy were performed only on 2 patients as part of a workshop.

All the procedures were done by general gynaecologists but for the 3 procedures which were done in a workshop, where in the surgeries were done by gynaecologists with some training in urology.

\section{Intra op complications}

Haemorrhage was seen in only one patient undergoing sacrospinous fixation which was managed conservatively with subsequent transfusion. Ureteric injury was not encountered in any case. 2 cases of bladder injury were noted during anterior colporrhaphy step of traditional repair procedure which were recognised on table and repaired immediately.

\section{Immediate post op complications}

SSF is sometimes followed immediately with severe postoperative gluteal pain that runs down the posterior surface of the affected leg and is most likely due to a pudendal nerve injury. ${ }^{6}$ No such complication was seen in any of the 26 patients who underwent SSF.

Sutures that are placed too close to the ischial spine risk injury to the pudendal nerves and the sciatic nerve. If the patient has evidence of nerve injury in the form of pain, numbness, tingling etc. immediate reoperation with removal of those sutures impinging on the nerve is warranted. None of our patients had such a complication.

Approximately $10 \%$ to $15 \%$ of patients have transient moderate to severe buttock pain on the side of the sacrospinous suspension and is usually self-limiting. ${ }^{6}$ $16 \%$ of our patients (4/24) had complaints of buttock pain. Reassurance and anti-inflammatory agents were used and all of them were relieved of this pain in 6 weeks.

ICU admissions were required in 5 patients who were monitored in ICU for 2-3 days of which only 1 was for intra-op complications (bleeding), while others were in ICU for co-morbid conditions.

Febrile morbidity which is a common complication with any surgery was seen in 8 patients and bladder symptoms of dysuria and pain were seen in 4 patients who also had evidence of UTI. Retention of urine was complained of by 2 patients who were conservatively managed.

In majority of vaginal repair cases catheter was removed in 48-72 hours with 2 of them requiring re-catheterization for 5 more days.

Mean duration of hospital stay was 7 days although most of them were fit for discharge by 4 days as patients hailed from distant locations and preferred to stay longer in the hospital.

\section{Success of treatment}

Patient-reported outcomes, including patient-reported success rates and relief of presenting symptoms, were the primary outcome assessed. Symptomatic relief was noted in $95 \%$ of the patients who were available for follow up at 6 weeks. Only 12 patients were available for follow up for 6 months who were satisfied with the outcome. Majority of the patients were lost to long term follow up.

\section{DISCUSSION}

The pathophysiology behind vault prolapse has been studied for a long time and several mechanisms have been hypothesized to explain the same. Attempts at identifying risk factors that predispose to vault prolapse have been going on relentlessly.

Descent of vaginal vault to the hymenal ring is often the time when patients complain of vaginal bulge. However lesser degree of descent may also be symptomatic. ${ }^{7}$ Use of standardized terminology and classification systems enables assessment of outcomes for individual women and facilitates the comparison of results for audit and research purposes. POPQ system is commonly used for grading and comparing the outcomes in pelvic organ prolapse but the study period saw the usage of POP Q 
system only in the last 3 years and hence was not considered in the study.

Performing preoperative urodynamic testing with and without prolapse reduction in continent women undergoing POP surgery does not always predict postoperative stress incontinence. Clinical assessment remains the most important assessment tool. ${ }^{6}$ Prophylactic treatment for women thought to have occult stress urinary incontinence (SUI) will result in unnecessary treatment for some women.

Choice of surgery depends on various factors. They are Vaginal length and width, co-existent anterior and posterior wall prolapse, Surgeon's expertise, age of the patient, other co morbid conditions and her fitness for surgery and whether she is sexually active or not. Some cases where past abdominal surgery could have caused lot of adhesions, vaginal route is preferred and with previous vaginal repair, abdominal route helps to maintain the depth of the vagina. Study by Malathi dalal et al reports doing only sacrspinous fixation in all the 35 cases. $^{8}$

The incidence of vaginal vault prolapse was $11.6 \%$ (14/120 patients) when hysterectomy had been performed for genital prolapse and $1.8 \%$ (6/328) when hysterectomy had been performed for other benign diseases. In the latter group (328 patients), all the cases of vault prolapse developed after abdominal hysterectomy: the incidence was $2 \%$ (6/308 patients); no case (0/20 patients) of vault prolapse developed after vaginal hysterectomy that had been performed on patients without genital prolapse. ${ }^{9}$

Elkins et al compared high uterosacral suspensions with sacrospinous fixation. Total vaginal length from introitus to vaginal cuff, recorded at rest 6-12 weeks postoperatively, measured $10.2 \mathrm{~cm}$ and $8.3 \mathrm{~cm}$, respectively. They also studied anatomical measurements in 12 fresh, unembalmed cadavers to compare the anatomical aspects of sacrospinous fixation and high uterosacral fixation. Sacrospinous ligament fixation resulted in a deeper posterior vaginal deviation with a broader angle of deflection than seen with high uterosacral suspension. The former measured 47.1 degrees (range 42-50 degrees) and the latter 35 degrees (range 25-40 degrees). The sacrospinous fixation also caused lateral displacement of the vagina from the midpelvis to the mid-ligament by 45 degrees (range $38-58$ degrees). It seems, therefore, that uterosacral suspension achieves a better anatomical result than sacrospinous fixation, especially in restoring the vaginal axis. ${ }^{10}$

Sacrospinal fixation was found to very effective and complications were very rare. Only one case of hemorrhage and 4 cases of transient buttock pain out of 24 in the series was reported by Maher CE et al. ${ }^{11}$ Our series of 26 cases also has shown 8 cases of buttock pain, one with bleeding which was significant which was transient. 2 cases of transient febrile morbidity were encountered. Febrile morbidity was the commonest complication encountered in the study by Morley GN et al. $^{12}$

Though Laparoscopic approach is equally effective with many advantages like less morbidity, less hospital stay, enhanced view - more anatomic repair, less scarring, requires high level of expertise, longer operating time and in our series it was done only during workshops operated by people with expertise in laparoscopic surgeries wherein no complications were encountered. ${ }^{13,14}$

Conventional repair alone was done in (12 out of 56) mostly during the early period of this study and 2 cases of intra operative bladder injuries were noticed immediately repaired by the operating surgeon. But recommendations go to say that conventional repair alone with no fixation procedures is inadequate for vault prolapsed. ${ }^{15}$

There are now level B recommendations for preventive measures like McCall culdoplasty at the time of vaginal hysterectomy, Suturing the cardinal and uterosacral ligaments to the vaginal cuff at the time of hysterectomy in both abdominal and vaginal hysterectomies, sacrospinous fixation (SSF) at the time of vaginal hysterectomy when the vault descends to the introitus during closure. However in our study almost all patients had no detailed records of the hysterectomy procedure and thus could not be analyzed.

Utero-sacral and cardinal ligaments are often very weak, and sometimes not even identifiable. The traditional vaginal repair of the vault prolapse hence involves over enthusiastic anterior and posterior colporhaphy and aggressive perineorrhaphy often resulting in much narrowing of the vaginal canal and the introitus. Hence traditional repair is not routinely recommended for vault prolapse. But in the present study traditional repair was done in the earlier part of the study period for some patients.

Sacrospinous fixation, abdominal sacrocolpopexy, was more frequently done and HUSLS done only for demonstration in a workshop.

Hemorrhage during SSF can result from injury to the hypogastric venous plexus, inferior gluteal vessels, and internal pudendal vessels and from overzealous dissection or inappropriate needle passage through the sacrospinous ligament. ${ }^{1}$

Complications of HUSLS include most commonly ureteric injury, the incidence of which can be as high as $10.9 \%$, bladder injury, urinary tract infection, blood transfusion and small bowel injury. ${ }^{6}$ Placing the sutures into the deep dorsal aspect of the ligament is reported to reduce the incidence of ureteric injury, especially in the laparoscopic approach. Suture erosion was also noted with permanent sutures. 
Quality of life questionnaires are helpful in patient centre assessment and care. They require additional resources and time. ${ }^{6}$ These were unavailable and not used for our patients.

\section{CONCLUSION}

Prevention is better than re-operation. Primary selection of cases and appropriate procedure are the key to success. McCall's culdoplasty at the time of vaginal hysterectomy is recommended. Sacrospinous fixation and abdominal sacrocolpopexy (open/laparoscopic) are both effective with minimal complications. HUSLS presently is not recommended for routine practise. ${ }^{16}$

Choosing the route of surgery, procedure depends on surgeon's expertise, patient's requirements, condition of the tissues and past surgical interventions.

Funding: No funding sources

Conflict of interest: None declared

Ethical approval: Not required

\section{REFERENCES}

1. Te linde's. Operative gynecology $10^{\text {th }}$ ed Lippincott Raven, Philadelphia; 1997:911.

2. Berek. Novak's Gynecology $15^{\text {th }}$ edition Lippincott Williams and Wilkins, Philadelphia; 2012.

3. Parikh M. Vault Prolapse. J Obstet Gynecol India. 2005;1;20-1.

4. Maher C, Feiner B, Baessler K, Schmid C. Surgical management of pelvic organ prolapse in women. Cochrane Database Syst Rev. 2004;(4):CD004014.

5. Dällenbach P. To mesh or not to mesh: a review of pelvic organ reconstructive surgery. Int J Womens Health. 2015;7:331-43.

6. Hardiman PJ, Drutz HP. Sacrospinous vault suspension and abdominal colposacropexy: success rates and complications. Am J Obstet Gynecol. 1996;17:612-6.

7. Barrington JW, Edwards G. Post hysterectomy vault prolapse. Int Urogynecol J Pelvic Floor Dysfunct. 2000;11:241-5.

8. Malti D, Ragini NV, Tejal SS, Heena GC. Sacrospinous colpopexy for vault suspension. J Obstet Gynecol India. 2006;56(3):247-9.

9. Marchionni M, Bracco GL, Checcucci V, Carabaneanu A, Coccia EM, Mecacci F, et al. True incidence of vaginal vault prolapse. Thirteen years of experience. J Reprod Med. 1999;44(8):679-84.

10. Elkins TE, Hopper JB, Goodfellow K, Gasser R, Nolan TE, Schexnayder MC. Initial report of anatomic and clinical comparison of the sacrospinous ligament fixation to the high McCall culdoplasty for vaginal cuff fixation at hysterectomy for uterine prolapse. Journal of Pelvic Surgery. 1995;1:12-7.

11. Maher CF, Qatawneh AM, Dwyer PL, Carey MP, Cornish A, Schluter PJ. Abdominal sacral colpopexy or vaginal sacrospinous colpopexy for vaginal vault prolapse: a prospective randomized study. Am J Obstet Gynecol. 2004;190:20-6.

12. Morley GN, DeLancey JO. Sacrospinous ligament fixation for eversion of vagina. Am $\mathrm{J}$ Obstet Gynecol. 1988;158:872-9.

13. Margossian H, Walters MD, Falcone $\mathrm{T}$. Laparoscopic management of pelvic organ prolapsed. Eur I Obstet Gynecol Reprod Biol. 1999;85:57-62.

14. Higgs PJ, Chua HL, Smith AR. Long term review of laparoscopic sacrocolpopexy. BJOG. 2005;112(8):1134-8.

15. Toozs-Hobson P, Boos K, Cardozo L. Management of vaginal vault prolapse. BJOG. 1998;105:13-7.

16. RCOG green top guidelines 46,2015 . Available at https://www.rcog.org.uk/globalassets/documents/gui delines/gtg-46.pdf.

Cite this article as: Jois SK, Rao LG. Vault prolapse - a decade's experience. Int J Reprod Contracept Obstet Gynecol 2016;5:2989-94. 